\title{
Thrombotic Microangiopathy Associated with Gemcitabine in Non-Small Cell Lung Cancer: A Case Report
}

\author{
Tadahiro Kuribayashi $^{a}$ Keiichi Fujiwara ${ }^{a}$ Kiriko Onishi ${ }^{a}$ \\ Sho Mitsumune ${ }^{a}$ Yuki Takigawa ${ }^{\text {a }}$ Hiromi Watanabe $^{a}$ \\ Kenichiro Kudo ${ }^{a}$ Akiko Sato $^{a} K$ Ken Sato ${ }^{a}$ Masashi Kitagawa ${ }^{b}$ \\ Kosuke Ota $^{b}$ Yoko Shinno $^{c}$ Takuo Shibayama ${ }^{a}$ \\ aDepartment of Respiratory Medicine, National Hospital Organization Okayama Medical \\ Center, Okayama, Japan; 'bepartment of Nephrology, National Hospital Organization \\ Okayama Medical Center, Okayama, Japan; 'Department of Pathology, National Hospital \\ Organization Okayama Medical Center, Okayama, Japan
}

\section{Keywords}

Thrombotic microangiopathy · Non-small cell lung cancer · Gemcitabine · Autopsy ·

Plasmapheresis

\begin{abstract}
A 69-year-old man with refractory lung adenocarcinoma was treated with gemcitabine and vinorelbine. Dyspnea and hypertension developed after the 17th cycle of chemotherapy. Laboratory findings revealed intravascular hemolysis and renal dysfunction. Thrombotic microangiopathy (TMA) was confirmed by renal biopsy. Antihypertensive and steroid therapies were ineffective. After plasmapheresis, intravascular hemolysis and renal dysfunction gradually improved. However, the disease progressed, and he died 6 months after TMA diagnosis. Autopsy revealed similar pathological findings to those of the renal biopsy. It is important to discontinue gemcitabine at the onset of TMA and consider TMA when using gemcitabine for long periods.
\end{abstract}

\section{Karger $\stackrel{2}{\circ}$}


Kuribayashi et al.: Gemcitabine-Associated Thrombotic Microangiopathy

\section{Introduction}

Gemcitabine is a pyrimidine antimetabolite that acts against solid tumors and has been shown to be effective against non-small cell lung cancer (NSCLC). The major adverse side effects of gemcitabine include hematologic toxicity, peripheral edema, asthenia, and flu-like symptoms [1]. Additionally, there have been few reports of renal failure caused by thrombotic microangiopathy (TMA) [2].

TMA is a microvascular occlusive disorder characterized by the clinical presentation of thrombocytopenia, hemolytic anemia, and organ injury. TMA is classified into primary, secondary, or infection associated TMA. Secondary TMA is induced by autoimmune conditions, severe hypertension, pregnancy, malignancy, bone marrow transplantation, and drug therapy. In a previous review, drugs causing TMA included tacrolimus, cyclosporine, interferons, bevacizumab, sunitinib, gemcitabine, mitomycin, quinine, and ticlopidine [3].

We previously reported a case of bilateral testicular metastases from lung adenocarcinoma that showed an objective response to nivolumab [4]. After the 23rd cycle of nivolumab treatment, the disease progressed, and the patient received combination chemotherapy consisting of docetaxel/ramucirumab and subsequent biweekly gemcitabine/vinorelbine treatment. Thereafter, he developed TMA, possibly induced by gemcitabine. Thus, we herein describe an additional report of a case of gemcitabine-induced TMA confirmed by renal biopsy and autopsy.

\section{Case Report}

A 69-year-old man was admitted to our hospital with progressive hypertension and renal dysfunction in May 2020. He was diagnosed with locally advanced stage IIIA lung adenocarcinoma in December 2016 [4]. Driver gene mutation tests were negative, and PD-L1 was expressed in $<1 \%$ of cancer cells in the tumor. He had a history of diabetes mellitus and gastric ulceration and had been a smoker between 20 and 65 years of age. He was originally treated with combined chemotherapy consisting of carboplatin, paclitaxel, and concurrent thoracic radiation therapy. In August 2017, bilateral testicular and adrenal metastases were detected; thus, the patient was diagnosed with recurrent lung cancer. He received 3 lines of chemotherapeutic regimens: 5 cycles of carboplatin plus pemetrexed, 23 cycles of nivolumab, and 6 cycles of docetaxel plus ramucirumab as first-, second-, and third-line therapy, respectively. In addition, we performed a left orchiectomy for testicular metastases in November 2017. In August 2019, the disease was refractory to docetaxel and ramucirumab treatment; thus, we began subsequent chemotherapy consisting of vinorelbine $\left(25 \mathrm{mg} / \mathrm{m}^{2}\right)$ and gemcitabine $\left(750 \mathrm{mg} / \mathrm{m}^{2} ; 1,000 \mathrm{mg} /\right.$ body) scheduled every 2 weeks as salvage therapy.

Five days after the 17 th cycle (cumulative dose of gemcitabine, $12,750 \mathrm{mg} / \mathrm{m}^{2}$ ), he presented with dyspnea on exertion and anorexia and was admitted to our hospital. Upon physical examination, his vital signs were normal except for massive hypertension (157/99 mm Hg). His blood pressure was low at approximately 100/60 mm Hg before treatment with vinorelbine and gemcitabine. Laboratory findings showed intravascular hemolysis (hemoglobin, $6.8 \mathrm{~g} / \mathrm{dL}$; lactate dehydrogenase (LDH), $709 \mathrm{U} / \mathrm{L}$; haptoglobin, $<10 \mathrm{mg} / \mathrm{dL}$; schistocytes in a blood smear; negative Coombs test), thrombocytopenia $\left(10.0 \times 10^{4} / \mu \mathrm{L}\right)$, and renal dysfunction (serum creatinine level, $1.20 \mathrm{mg} / \mathrm{dL}$; proteinuria, $1.63 \mathrm{~g} /$ day) (Table 1). He had no history of infectious disease. His ADAMTS-13 activity was normal, and autoantibody tests were negative. Computed tomography scans showed

\section{Karger' ${ }^{\prime \prime}$}


Table 1. Laboratory findings on admission to our hospital

\begin{tabular}{|c|c|c|c|c|c|c|c|c|}
\hline \multicolumn{3}{|c|}{ Hematology } & \multirow{2}{*}{$\begin{array}{l}\text { LDH } \\
\text { ALP }\end{array}$} & \multirow{2}{*}{$\begin{array}{l}709 \\
206\end{array}$} & \multirow{2}{*}{$\frac{\mathrm{U} / \mathrm{L}}{\mathrm{U} / \mathrm{L}}$} & \multirow{2}{*}{$\frac{\mathrm{CH}_{50}}{\mathrm{CEA}}$} & \multirow{2}{*}{$\begin{array}{l}50.8 \\
6.8\end{array}$} & \multirow{2}{*}{$\frac{\mathrm{U} / \mathrm{mL}}{\mathrm{ng} / \mathrm{mL}}$} \\
\hline WBC & 8,300 & $/ \mu \mathrm{L}$ & & & & & & \\
\hline Seg & 83.3 & $\%$ & $\gamma$-GTP & 38 & $\mathrm{U} / \mathrm{L}$ & SLX & 33 & $\mathrm{U} / \mathrm{mL}$ \\
\hline Mon & 7.4 & $\%$ & $\mathrm{TP}$ & 5.1 & $\mathrm{~g} / \mathrm{dL}$ & $\mathrm{RF}$ & 13 & $\mathrm{U} / \mathrm{mL}$ \\
\hline Lym & 9.0 & $\%$ & ALB & 3.2 & $\mathrm{~g} / \mathrm{dL}$ & ANA & $<\times 40$ & \\
\hline Eos & 0.1 & $\%$ & CRE & 1.20 & $\mathrm{mg} / \mathrm{dL}$ & PR3-ANCA & $<1.0$ & $\mathrm{U} / \mathrm{mL}$ \\
\hline Bas & 0.2 & $\%$ & BUN & 28 & $\mathrm{mg} / \mathrm{dL}$ & MPO-ANCA & $<1.0$ & $\mathrm{U} / \mathrm{mL}$ \\
\hline $\mathrm{RBC}$ & 222 & $\times 10^{4} / \mu \mathrm{L}$ & $\mathrm{Na}$ & 144 & $\mathrm{mEq} / \mathrm{L}$ & ADAMTS-13 activity & 0.67 & $\mathrm{U} / \mathrm{mL}$ \\
\hline $\mathrm{Hgb}$ & 6.8 & $\mathrm{~g} / \mathrm{dL}$ & $\mathrm{K}$ & 4.1 & $\mathrm{mEq} / \mathrm{L}$ & ADAMTS-13 inhibitor & $<0.5$ & $\mathrm{BU} / \mathrm{mL}$ \\
\hline Hct & 20.5 & $\%$ & $\mathrm{Cl}$ & 110 & $\mathrm{mEq} / \mathrm{L}$ & Haptoglobin & $\leqq 10$ & $\mathrm{mg} / \mathrm{dL}$ \\
\hline PLT & 10.0 & $\times 10^{4} / \mu \mathrm{L}$ & \multicolumn{2}{|c|}{ serology } & & Direct coombs test & Negative & \\
\hline $\mathrm{MCV}$ & 92.3 & $\mathrm{fL}$ & CRP & 1.32 & $\mathrm{mg} / \mathrm{dL}$ & urinalysis & & \\
\hline PT & 93 & $\%$ & HbA1c & 5.2 & $\%$ & Glucose & $(-)$ & \\
\hline APTT & 29.1 & sec & IgG & 439 & $\mathrm{mg} / \mathrm{dL}$ & Blood & $(3+)$ & \\
\hline D-dimer & 2.5 & $\mu \mathrm{g} / \mathrm{dL}$ & $\operatorname{IgA}$ & 68 & $\mathrm{mg} / \mathrm{dL}$ & Protein & 687.2 & $\mathrm{mg} / \mathrm{dL}$ \\
\hline \multicolumn{2}{|c|}{ biochemistry } & & $\operatorname{IgM}$ & 27 & $\mathrm{mg} / \mathrm{dL}$ & CRE & 167.1 & $\mathrm{mg} / \mathrm{dL}$ \\
\hline T-Bil & 0.8 & $\mathrm{mg} / \mathrm{dL}$ & $\mathrm{C} 3$ & 109 & $\mathrm{mg} / \mathrm{dL}$ & NAG & 54.2 & $\mathrm{U} / \mathrm{L}$ \\
\hline AST & 26 & $\mathrm{U} / \mathrm{L}$ & $\mathrm{C} 4$ & 29 & $\mathrm{mg} / \mathrm{dL}$ & $\beta_{2} \mathrm{MG}$ & 23 & $\mu \mathrm{g} / \mathrm{L}$ \\
\hline ALT & 13 & $\mathrm{U} / \mathrm{L}$ & $\mathrm{C} 1 \mathrm{q}$ & $\leqq 1.5$ & $\mu \mathrm{g} / \mathrm{mL}$ & & & \\
\hline
\end{tabular}

WBC, white blood cell; RBC, red blood cell; Hgb, hemoglobin; Hct, hematocrit; PLT, platelet; MCV, mean corpuscular volume; PT, prothrombin time; APTT, activated partial thromboplastin time; T-Bil, total bilirubin; AST, aspartate aminotransferase; ALT, alanine aminotransferase; $\gamma$-GTP, $\gamma$-glutamyl transpeptidase; LDH, lactate dehydrogenase; ALP, alkaline phosphatase; TP, total protein; ALB, albumin; CRE, creatinine; BUN, blood urea nitrogen; CRP, C-reactive protein; HbA1c, hemoglobin A1c; CEA, carcinoembryonic antigen; SLX, sialyl Lewis-x antigen; IgG, immunoglobulin G; IgA, immunoglobulin A; IgM, immunoglobulin M; RF, rheumatoid factor; ANA, antinuclear antibody; PR3-ANCA, proteinase 3-anti-neutrophil cytoplasmic antibody; MPO-ANCA, myeloperoxidase-anti-neutrophil cytoplasmic antibody; ADAMTS-13, a disintegrinlike and metalloproteinase with thrombospondin type 1 motifs 13 ; NAG, N-acetyl- $\beta$-D-glucosaminidase; $\beta_{2} \mathrm{MG}, \beta_{2}$ microglobulin.

increased bilateral pleural effusion and pericardial effusion, but no changes in lung, lymph node, and adrenal metastases (Fig. 1). Cytopathologic examination of the pleural effusion revealed no malignant cells.

On the 4th day of admission, a renal biopsy was performed. Pathological findings of the biopsied specimen showed diffuse and global duplication of the glomerular basement membranes due to subendothelial swelling. Light microscopy did not reveal an increase in mesangial cells (Fig. 2a, b). Immunofluorescence staining showed $\operatorname{IgG}( \pm), \operatorname{IgA}(-), \operatorname{Ig} M$ $(+)$, C3 $(-)$, C1q $(-)$, and fibrinogen $(-)$. In electron microscopy, glomeruli showed endocapillary cell proliferation and endothelial swelling. Electron dense deposits were not observed in the glomeruli (Fig. 2c). Thus, the renal biopsy findings were consistent with those found in TMA patients. Considering all plausible causes, gemcitabine seemed to be responsible for the development of TMA, and further chemotherapy was omitted. Subsequently, he received antihypertensive therapy with a calcium channel blocker, loop diuretics, and steroid therapy (30 mg of intravenous prednisolone) (Fig. 3). However, renal dysfunction gradually worsened, and LDH levels increased. On the 47th day, he 


\section{Case Reports in Oncology}
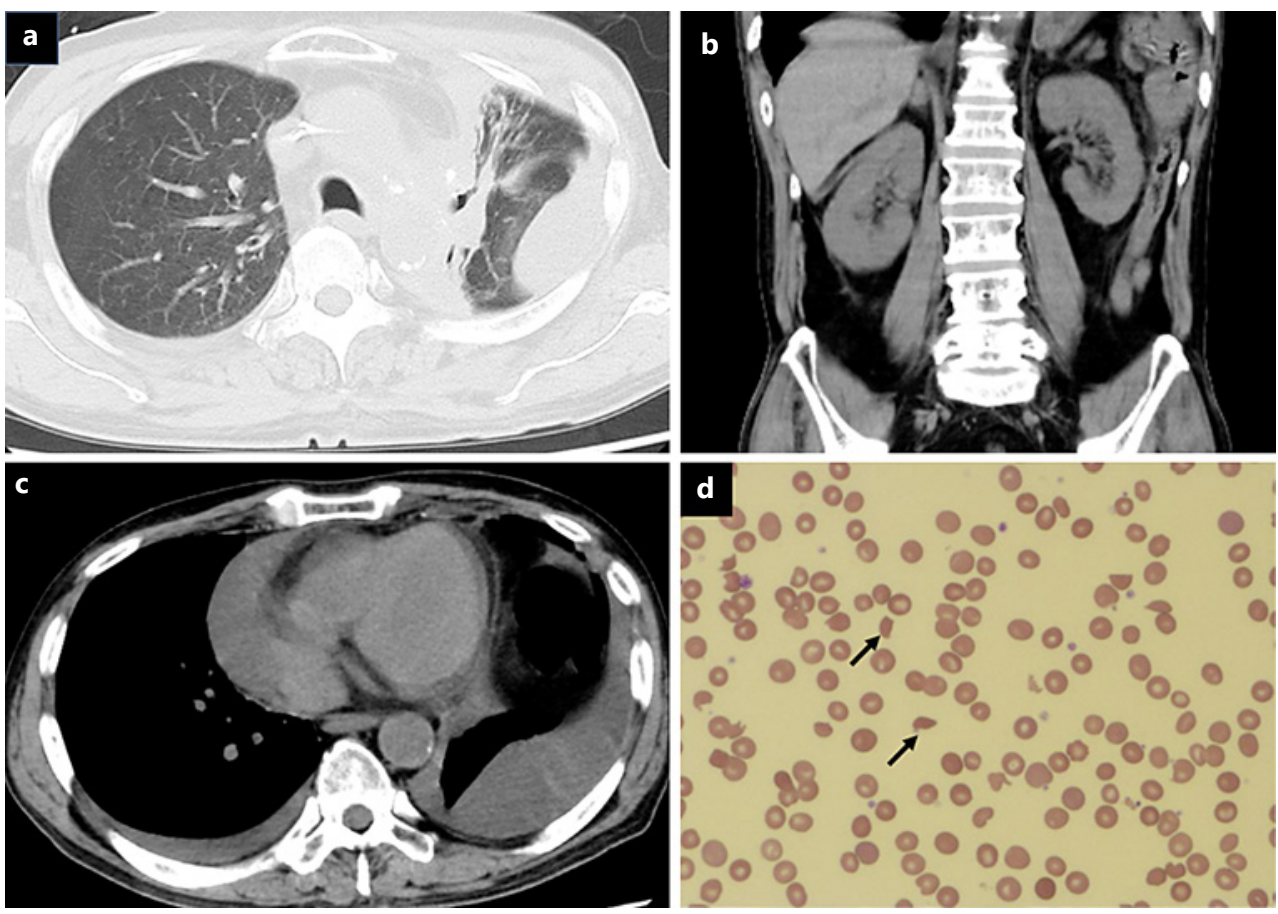

Fig. 1. Computed tomography scans obtained upon admission to our hospital (a-c). The primary lesion in the left upper lobe of the lung shows no change (a). Pericardial effusion and bilateral pleural effusion are observed (b). Neither kidney shows abnormal morphological findings (c). A peripheral blood smear showing many schistocytes, indicated by arrows (d).

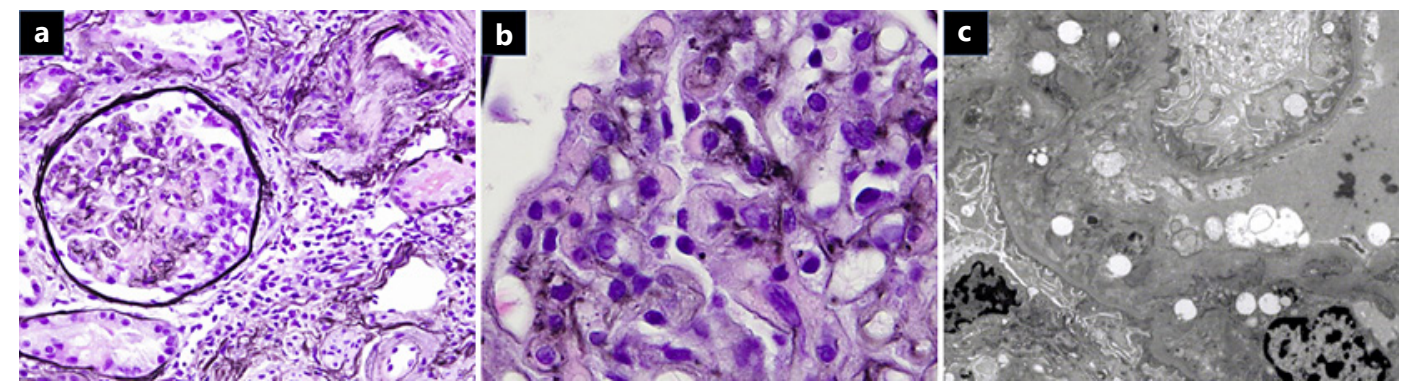

Fig. 2. Pathological findings of the biopsied specimen obtained from the left kidney with periodic acid-methenamine silver stain in light microscopy $(\mathbf{a}, \mathbf{b})$ and with electron microscopy (c) showing thrombotic microangiopathy. Diffuse and global duplication of the glomerular basement membrane and subendothelial swelling are observed. Electron dense deposits are not observed in glomeruli.

underwent 4 sessions of plasmapheresis. After plasmapheresis, nephrotic-range proteinuria persisted, but schistocytes in the blood smear disappeared, and LDH and serum creatinine levels decreased.

His general condition gradually deteriorated due to the progression of the lung cancer. He died in September 2020. An autopsy was performed after securing his family's permission. Metastases of cancer were found in both lungs, mediastinal lymph nodes, adrenal glands, liver, and the remaining right testicle. Both kidneys were atrophied (right, $75 \mathrm{~g}$; left, $75 \mathrm{~g}$ ). In addition to arteriosclerotic changes, the pathological findings of the autopsy were consistent with TMA, and they were similar to those of the renal biopsy. 


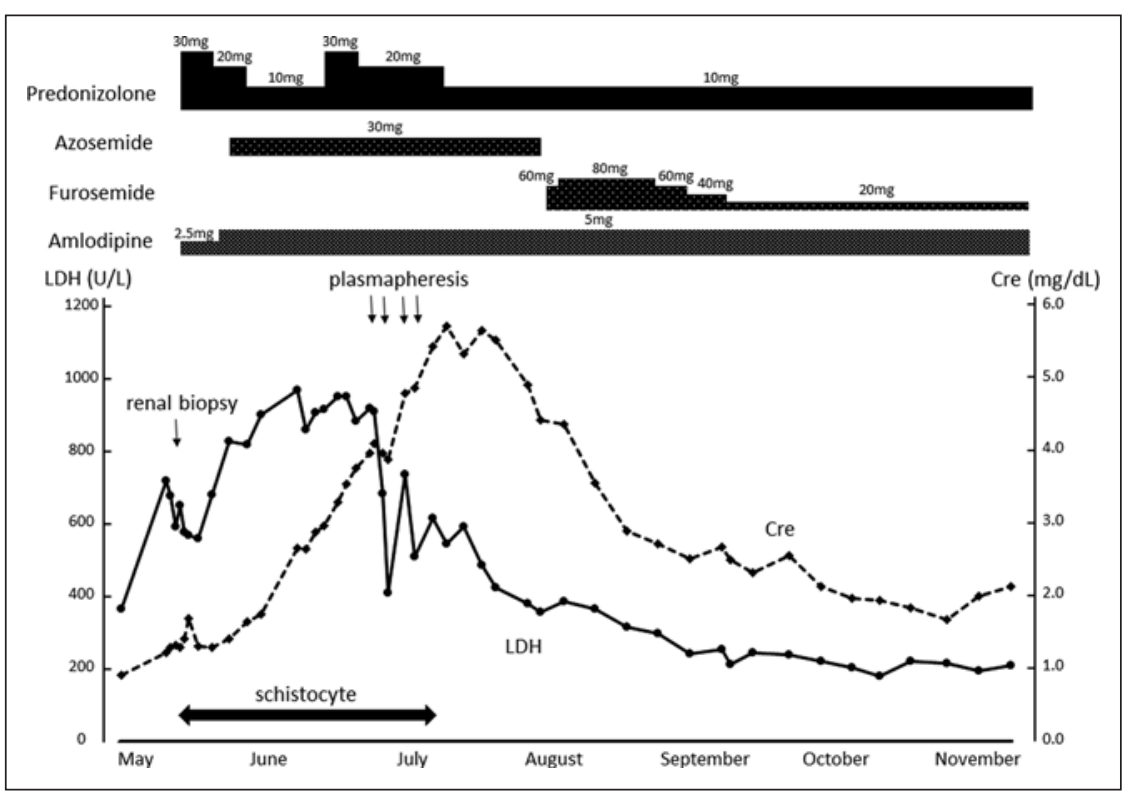

Fig. 3. The clinical course of thrombotic microangiopathy in the present case. After admission, LDH and Cre levels worsened and schistocytes appeared. Plasmapheresis was effective in decreasing LDH and Cre, although antihypertensive therapy and steroids were not. LDH, lactate dehydrogenase; Cre, serum creatinine level.

\section{Discussion}

In the present case, the patient developed TMA after salvage combination chemotherapy consisting of gemcitabine and vinorelbine. Recently, this combined chemotherapy has not been used in the treatment of NSCLC, except in late-line chemotherapy. However, a previous trial indicated that this regimen is less toxic (e.g., vomiting, appetite, and hair loss) than cisplatinbased regimens [5]. In our case, he desired the treatment with less gastrointestinal toxicity. Although the evidence is poor, we consider chemotherapy consisting of gemcitabine and vinorelbine for NSCLC patients who desire anticancer treatment during late-line chemotherapy.

TMA has been reported to be caused by several anticancer drugs, such as gemcitabine, ramucirumab [6], docetaxel [7], and nivolumab [8]. However, in the reports, these anticancer agents were used immediately before the development of TMA. Additionally, there have been no published reports of vinorelbine-induced TMA. Therefore, it is unlikely that ramucirumab, docetaxel, and vinorelbine were the causes of TMA in this case. Gergi et al. [8] reported nivolumab-induced TMA 12 weeks after the initiation of treatment. In their report, immune checkpoint inhibitor-related TMA improves within several days by the treatment with prednisone, plasmapheresis, and rituximab. Although treatment duration before kidney injury is variable in immune checkpoint inhibitor treatment [9], nivolumab involvement in the occurrence of TMA seemed to be also unlikely in our case.

The incidence of gemcitabine-associated TMA is $0.015-1.4 \%$ [10]. The median development time of a TMA syndrome after the initiation of gemcitabine ranged from 7 to 8 months, with a cumulative dose ranging from 6 to $56 \mathrm{~g} / \mathrm{m}^{2}$ [2,11]. Daviet et al. [11] reported that 119 cases of gemcitabine-associated TMA occurred mainly in the treatment of pancreatic cancer (52.9\%) and lung cancer (12.6\%). There are only a few published reports in which TMA was proven pathologically by renal biopsy or autopsy in lung cancer [12]. In our case, the patient was diagnosed with TMA 8.5 months after the initiation of gemcitabine treatment with a cumulative dose of $12.75 \mathrm{~g} / \mathrm{m}^{2}$. Thus, we considered the possibility of gemcitabine-induced TMA. 
Humphreys et al. [2] reported that hypertension or exacerbation of existing hypertension preceded clinical TMA diagnosis in 7 of 9 patients. In addition, hypertension was the most common finding in 7 of 12 patients with TMA in a report by Fung et al. [13]. The severity of hypertension is correlated with poorer outcomes [14]. The patient's blood pressure was initially low but then increased 8.5 months after gemcitabine administration. It is important to perform a physical examination carefully and detect hypertension at an early stage in patients treated with gemcitabine to prevent aggravation.

The treatments for TMA include plasmapheresis and steroids, in addition to the discontinuation of gemcitabine and antihypertensive medications [10]. A previous study suggested that patients treated with plasmapheresis displayed comparable rates of remission, despite more severe acute kidney damage [11]. In contrast, Gore et al. [15] reported that the remission rate was higher without the use of plasmapheresis than that with its use, although there were differences in dialysis rate. Thus, it is unclear whether plasmapheresis is effective in the treatment of TMA. In the present case, gemcitabine discontinuation, antihypertensive treatment, and steroids were ineffective in treating TMA. However, LDH and serum creatinine levels decreased after plasmapheresis. Therefore, plasmapheresis seemed to prevent the progression of TMA and was somewhat effective for this patient.

In conclusion, we report an unusual adverse event of gemcitabine-induced TMA in a patient with NSCLC. Gemcitabine-induced TMA is relatively rare, and there has been no established treatment. It is extremely important to discontinue gemcitabine in the early stages of TMA onset in a clinical setting.

\section{Acknowledgment}

We would like to thank Editage (www.editage.com) for English language editing.

\section{Statement of Ethics}

Written informed consent for publication of this case report and any accompanying images was obtained from the patient's daughter. Case reports are granted an exemption from requiring ethical approval at the National Hospital Organization Okayama Medical Center due to the nature of reporting based on daily clinical practice.

\section{Conflict of Interest Statement}

The authors state that they have no conflicts of interest.

\section{Funding Sources}

The authors received no funds for this case report.

\section{Author Contributions}

All the authors contributed to this manuscript and approved the final version.

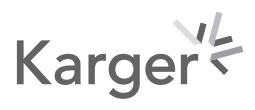




\section{Data Availability Statement}

All data generated or analyzed during this study are included in this article.

\section{References}

1 Abratt RP, Bezwoda WR, Falkson G, Goedhals L, Hacking D, Rugg TA. Efficacy and safety profile of gemcitabine in non-small-cell lung cancer: a phase II study. J Clin Oncol. 1994;12:1535-40.

2 Humphreys BD, Sharman JP, Henderson JM, Clark JW, Marks PW, Rennke HG, et al. Gemcitabine-associated thrombotic microangiopathy. Cancer. 2004;100:2664-70.

3 Brocklebank V, Wood KM, Kavanagh D. Thrombotic microangiopathy and the kidney. Clin J Am Soc Nephrol. 2018;13:300-17.

4 Ozeki T, Fujiwara K, Shimonishi A, Nishimura J, Okawa S, Takada K, et al. Bilateral testicular metastases from lung adenocarcinoma showing an objective response to nivolumab: a case report and review of the literature. Intern Med. 2019;58:3277-82.

5 Gridelli C, Gallo C, Shepherd FA, Illiano A, Piantedosi F, Robbiati SF, et al. Gemcitabine plus vinorelbine compared with cisplatin plus vinorelbine or cisplatin plus gemcitabine for advanced non-small-cell lung cancer: a phase III trial of the Italian GEMVIN Investigators and the National Cancer Institute of Canada Clinical Trials Group. J Clin Oncol. 2003;21:3025-34.

6 Yamada R, Okawa T, Matsuo K, Suzuki M, Mori N, Mori K. Renal-limited thrombotic microangiopathy after switching from bevacizumab to ramucirumab: a case report. BMC Nephrol. 2019;20:14.

7 Siau K, Varughese M. Thrombotic microangiopathy following docetaxel and trastuzumab chemotherapy: a case report. Med Oncol. 2010;27:1057-9.

8 Gergi M, Landry KK, Ades S, Barry M, Zakai NA, Herrera DA. Nivolumab-induced thrombotic thrombocytopenic purpura in a patient with anal squamous cell carcinoma: a lesson on hematologic toxicity from immunotherapy. Oncologist. 2020;25:1009-12.

9 Meraz-Muñoz A, Amir E, Ng P, Avila-Casado C, Ragobar C, Chan C, et al. Acute kidney injury associated with immune checkpoint inhibitor therapy: incidence, risk factors and outcomes. J Immunother Cancer. 2020;8: e000467.

10 Zupancic M, Shah PC, Shah-Khan F. Gemcitabine-associated thrombotic thrombocytopenic purpura. Lancet Oncol. 2007;8:634-41.

11 Daviet F, Rouby F, Poullin P, Moussi-Francès J, Sallée M, Burtey S, et al. Thrombotic microangiopathy associated with gemcitabine use: presentation and outcome in a national French retrospective cohort. $\mathrm{Br} J \mathrm{Clin}$ Pharmacol. 2019;85:403-12.

12 Suzuki Y, Kanazawa K, Kanai R, Tomita H, Saito M, Watanabe N, et al. A case of primary lung squamous cell carcinoma mimicking malignant mesothelioma producing granulocyte colony stimulating factor with chemotherapy (cisplatin and gemcitabine)-associated thrombotic thrombocytopenic purpura (TTP); An autopsy case report. Lung Cancer. 2019;136:105-8.

13 Fung MC, Storniolo AM, Nguyen B, Arning M, Brookfield W, Vigil J. A review of hemolytic uremic syndrome in patients treated with gemcitabine therapy. Cancer. 1999;85:2023-32.

14 Humphreys BD, Soiffer RJ, Magee CC. Renal failure associated with cancer and its treatment: an update. J Am Soc Nephrol. 2005;16:151-61.

15 Gore EM, Jones BS, Marques MB. Is therapeutic plasma exchange indicated for patients with gemcitabineinduced hemolytic uremic syndrome? J Clin Apher. 2009;24(5):209-14. 